DOI: https://doi.org/10.11144/Javeriana.umed59-2.cand

\title{
Opciones terapéuticas frente a especies de Candida resistentes a las equinocandinas
}

\section{Therapeutic Options in the Management of Echinocandin Resistant} Candida Species

Recepción: 29/11/2016 | Aceptación: 29/09/2017

\author{
Andrea Patricia Cortés Hidalgo ${ }^{a}$ \\ Pontificia Universidad Javeriana, Colombia \\ ORCID: http://orcid.org/0000-0002-7426-6181 \\ Oscar Hernando Roa Dueñas \\ Universidad de Boyacá, Colombia \\ ORCID: http://orcid.org/0000-0002-0752-2261 \\ Yardany Rafael MÉndez Fandiño \\ Universidad Pedagógica y Tecnológica de Colombia, \\ Colombia \\ ORCID: http://orcid.org/0000-0003-1528-2672 \\ Carlos A. Álvarez-Moreno \\ Universidad Nacional de Colombia, Colombia \\ ORCID: http://orcid.org/0000-0001-5419-4494
}

a Correspondencia: andrecortes29@gmail.com

\section{RESUMEN}

Introducción: La infección por levaduras del género Candida representa la causa más común de infecciones fúngicas invasivas. Su alta incidencia y la creciente resistencia frente a los azoles y, recientemente, a las equinocandinas ha generado la necesidad de buscar nuevas alternativas farmacológicas. Esta revisión presenta las principales alternativas farmacológicas en estudio frente a Candida resistente a equinocandinas. Métodos: Se buscó literatura referente al tema en las bases de datos Bireme, Clinical Key, Embase, Cochrane, Lilacs, Pubmed y Scopus. Se incluyeron 15 artículos en esta revisión. Resultados: Se exploran diferentes alternativas, incluyendo el aumento de dosis de las equinocandinas, su combinación con otros medicamentos y nuevos compuestos en estudio. Conclusión: A pesar de que las infecciones por Candida resistente a equinocandinas aún representan un desafío, dos alternativas farmacológicas se presentan como promisorias: la combinación con medicamentos existentes como el diclofenaco y nuevos compuestos que se encuentran actualmente en fase II de estudios clínicos. Palabras clave

equinocandinas; Candida; farmacorresistencia fúngica; antifúngicos.

\section{ABSTRACT}

Introduction: Candida yeasts infections represent the most common cause of invasive fungal infections. Its high incidence and increasing resistance to azoles and, recently, to echinocandins has generated the need to find new therapeutic options. This review presents the main pharmacological alternatives in research against echinocandins resistant Candida. Methods: A search was conducted in the databases of Bireme, Clinical Key, Embase, Cochrane, Lilacs, Pubmed and Scopus. 15 articles 
were included in this review. Results: Several alternatives are explored, including increased doses of echinocandins, combination with other drugs and new compounds under study. Conclusion: Although resistant Candida infections still represent a challenge, two pharmacological approaches show promise: The combination with existing medicaments such as diclofenac, and new compounds that are currently in Phase II of clinical trials.

Keywords

echinocandins; Candida; drug resistance; fungal; antifungal agents.

\section{Introducción}

La infección por levaduras del género Candida es la causa más común de infecciones fúngicas invasivas y sus manifestaciones clínicas van desde el compromiso mucocutáneo hasta la afectación invasiva, que incluso puede ser letal $(1,2)$.

Desde el punto de vista epidemiológico, la infección por especies de Candida constituye una importante causa de morbimortalidad, a pesar de los avances considerables en su tratamiento. Se encuentra entre los primeros cinco microrganismos comúnmente aislados en hemocultivos de pacientes hospitalizados (3) y la mortalidad global está entre un 30\% y un 60\%, con tasas de mortalidad atribuible del 25\% al 40\% $(3,4,5,6,7)$.

Con respecto a América Latina, la incidencia general de candidemia reportada en Brasil es de 2,49 casos por cada 1000 admisiones hospitalarias, y de 0,37 casos por cada 1000 pacientes/día (8). Ello es 3 a 15 veces mayor que la incidencia reportada en Norteamérica y Europa $(9,10)$. En Colombia, la incidencia reportada de infecciones por especies de Candida es de 2,3 casos por cada 1000 días de estancia en unidades de cuidado intensivo (11). Por otra parte, el aumento sostenido de la prevalencia de candidiasis invasiva, probablemente debido al incremento de pacientes inmunocomprometidos (12), y la aparición de resistencia al fluconazol, el medicamento de elección durante varios años, en especies previamente sensibles (v. g. Candida albicans) (13) o el incremento de especies con resistencia natural o disminuida (p. ej., C. glabrata o C. krusei) (14) determinó la necesidad de buscar tratamientos alternativos o superiores a los azoles.
Las equinocandinas se introdujeron por primera vez en el mercado farmacéutico en el 2001 como antifúngicos de amplio espectro $\mathrm{y}$ son un tratamiento efectivo frente a las infecciones invasivas por especies de Candida o Aspergillus sección Fumigatti $(15,16,17,18)$. Su utilidad radica en que pueden administrarse de forma empírica en pacientes críticamente enfermos, al actuar en las especies de Candida más frecuentes, incluyendo C. glabrata, C. krusei y C. auris, esta última de más reciente aparición; pero que dadas sus características de multirresistencia puede ser un problema serio en Colombia, donde esto ya se ha descrito $(19,20,21)$. Además, las equinocandinas presentan mínimas interacciones farmacológicas (22), escasos efectos adversos y no requieren ajuste de dosis en enfermos renales (1). Sin embargo, desde su introducción, en la práctica clínica se ha descrito su resistencia en especies de Candida. La literatura reporta tasas de prevalencia de resistencia entre $2,9 \%$ y 3,1\% (23) y en la mayoría de los casos corresponde a una resistencia adquirida postexposición a equinocandinas (24).

Entre los mecanismos de resistencia se encuentran tres de importancia: 1) una respuesta adaptativa frente al estrés, que aumenta el contenido de quitina en la pared celular y genera un crecimiento paradójico al administrar altas dosis de equinocandinas; 2) mutaciones en los genes FKS (genes relacionados con la codificación de la $\beta$-1,3-d-glucano sintasa) adquiridas, y 3) mutaciones FKS intrínsecas (14).

Por eso, la progresiva resistencia frente a las equinocandinas, asociada al incremento en su uso, la elevada incidencia de infecciones por Candida y la corresistencia a azoles y equinocandinas (25), ha generado la necesidad de estudiar los mecanismos de resistencia y buscar alternativas farmacológicas.

El objetivo de esta revisión narrativa es resumir y exponer las nuevas alternativas terapéuticas que se encuentran actualmente en estudio y que en un futuro podrían utilizarse para tratar las infecciones por Candida resistentes a equinocandinas. 


\section{Metodología}

Se realizó una búsqueda en la literatura disponible en las bases de datos Bireme, Clinical Key, Embase, Cochrane, LILACS, Pubmed y Scopus. Se realizaron 4 búsquedas en cada base de datos, utilizando los siguientes términos MeSH: Echinocandin Candida resistant, Anidulafungin Candida resistance (or resistant), Caspofungin Candida resistance (or resistant), Micafungin Candida Resistance (or resistant).

No se utilizó el idioma ni el tipo de artículo como filtro para evitar disminuir la sensibilidad, especialmente teniendo en cuenta que un número significativo de publicaciones sobre el tema son experimentos clínicos realizados en animales o in vitro.

Para esta revisión narrativa se seleccionaron todos los artículos que contenían información relevante sobre el tema, con fecha de publicación entre el 2006 y el 2016. Este filtro se seleccionó en vista de que la resistencia a equinocandinas es un tema reciente, con la comercialización de la primera equinocandina, Caspofungina, en el 2001. En total se incluyeron 15 artículos en esta revisión.

\section{Desarrollo}

Opciones de tratamiento en infecciones causadas por aislamientos de Candida resistente a equinocandinas.

\section{Aumento de dosis de equinocandinas}

Las equinocandinas presentan un excelente perfil de toxicidad, incluso al ser administradas en altas dosis (mayor de $8 \mathrm{mg} / \mathrm{kg} /$ día) (14). Por ello, se consideró la posibilidad de que el aumento de dosis fuera la solución frente a la creciente resistencia de Candida a las equinocandinas.

Inicialmente, por medio de un estudio realizado en ratones neutropénicos (26), se encontró que en la infección diseminada por C. glabrata con susceptibilidad reducida a caspofungina eran efectivas elevadas dosis de caspofungina y anidulafungina en reducir la carga fúngica renal (26). Sin embargo, en otro estudio llevado a cabo en ratones inmunocompetentes con infección invasiva por C. albicans (27) se observó una respuesta inconsistente frente a las altas dosis de caspofungina $(1-10 \mathrm{mg} / \mathrm{kg})$ en los ratones infectados con Candida resistente a caspofungina, y se encontró de $0 \%$ a $12 \%$ de sobrevida en los ratones infectados con especímenes más virulentos, y $80 \%$ en los ratones con especímenes menos virulentos. Debido a ello, se consideró que la respuesta a las altas dosis de caspofungina probablemente estaba relacionada con el grado de virulencia del espécimen (27).

La respuesta antifúngica al aumento de dosis de equinocandinas también se evaluó frente a C. glabrata (28). Se realizaron experimentos in vitro e in vivo, que incluyeron 4 especímenes clínicos de C. glabrata de tipo silvestre y, adicionalmente, un espécimen de referencia, sensible a caspofungina, y dos especímenes resistentes a equinocandinas con mutaciones conocidas en el FKS. Los especímenes resistentes a equinocandinas fueron altamente virulentos y ninguna dosis fue efectiva en los ratones infectados con los especímenes resistentes. Se confirmó así que el aumento de dosis no mejora la eficacia de la caspofungina (28).

\section{Nuevas combinaciones}

\section{Combinación con farnesol}

Farnesol, un compuesto orgánico, químicamente clasificado como sesquiterpeno alcohol (figura 1), se ha investigado por aumentar la susceptibilidad a antibióticos (29). Adicionalmente, Hornby y colaboradores (30) demostraron que este compuesto es sintetizado por C. albicans y se cree que en ciertas concentraciones puede ser citotóxico, al comprometer la síntesis de ergosterol, pues afecta la membrana celular fúngica y genera apoptosis celular (31).

Teniendo en cuenta lo anterior, un estudio realizado por Cordeiro y colaboradores (32) evaluó los efectos del farnesol en la susceptibilidad antifúngica de especímenes de 
Candida con resistencia in vitro a anfotericina $\mathrm{B}$, azoles y caspofungina. Mediante el uso de especímenes de C. albicans, C. parapsilosis y C. tropicalis, se demostró que al combinar el antifúngico con farnesol o preincubar los especímenes a concentraciones subinhibitorias de farnesol, se reducía la concentración inhibitoria mínima (CIM). La sinergia generada por la combinación de caspofungina con farnesol está posiblemente explicada por los efectos deletéreos en la pared celular causados por la caspofungina y la alteración de la biosíntesis de ergosterol producida por el farnesol (32).

Por tanto, se demostró sinergia al combinar el farnesol con medicamentos antifúngicos, lo cual mejora el efecto farmacológico en especímenes previamente resistentes. Sin embargo, aún hacen falta estudios que profundicen en el efecto y el mecanismo de acción del farnesol.

Figura 1.Estructura química de los nuevos fármacos con propiedades antifúngicas.
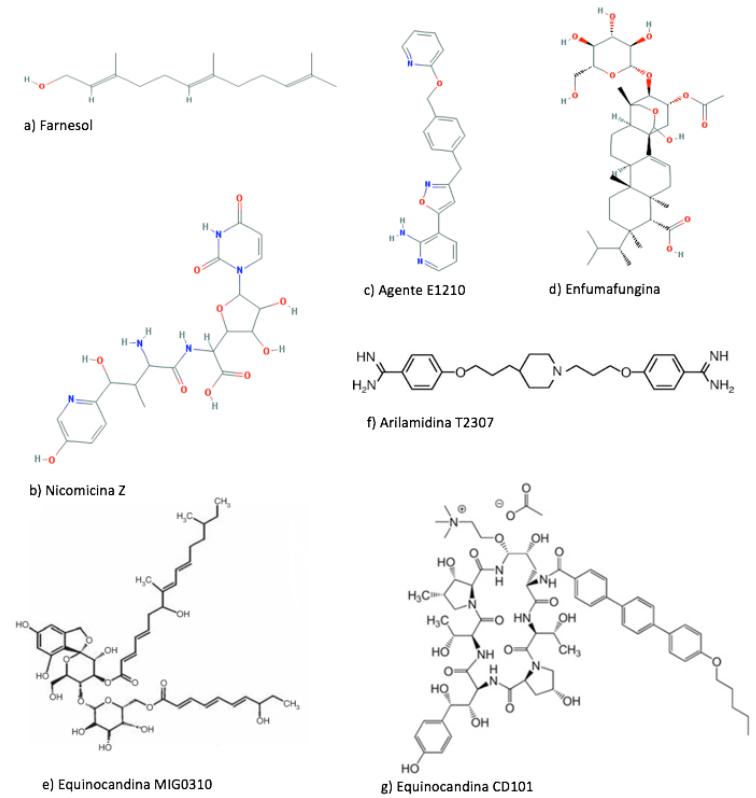

Tomado de: a) http://pubchem.ncbi.nlm.nih.gov/ compound/445070; b) https://pubchem.ncbi.nlm .nih.gov/compound/353481\#section=Top; c) h ttps://pubchem.ncbi.nlm.nih.gov/compound/167 19049\# section=Top; d) https://pubchem.ncbi.n lm.nih.gov/compound/25229651\#section=Top; e) http://onlinelibrary.wiley.com/doi/10.1111/jam .12493/epdf; f) http://www.ncbi.nlm.nih.gov/pm c/articles/PMC2292552/; g) http://www.nature.c om/ja/journal/vaop/ncurrent/full/ja201689a.html

\section{Combinación con diclofenaco}

Se desconoce el mecanismo de acción de este medicamento antinflamatorio no esteroideo en los hongos $(33,34)$. En el ámbito clínico, el diclofenaco se utiliza ampliamente como analgésico, debido a su acción sobre las isoenzimas ciclooxigenasas COX-1 y COX-2, las cuales se encuentran involucradas en la producción de prostaglandinas (35). Curiosamente, se ha observado que C. albicans también produce prostaglandinas (33) y que con la administración de inhibidores de la ciclooxigenasa disminuye esta producción y la viabilidad de la levadura (36). Por esa razón, se ha considerado que al ser un medicamento que actúa en la vía de las prostaglandinas podría ser una estrategia potencial terapéutica antifúngica (37).

Al combinar diclofenaco y caspofungina en especímenes de C. albicans, Bink y colaboradores (34) encontraron que in vitro los especímenes que crecían en presencia de diclofenaco tenían mayor susceptibilidad a la caspofungina. In vivo, los ratones, con un catéter subcutáneo, infectados con C. albicans y tratados con diclofenaco y caspofungina, tenían menos biopelícula de Candida que aquellos tratados únicamente con diclofenaco o caspofungina. Incluso lo anterior se demostró en el espécimen con mutación resistente a caspofungina. De esta manera se evidenció la sinergia entre el diclofenaco y la caspofungina, que probablemente esté relacionada con el aumento de la permeabilidad de las membranas celulares de C. albicans, inducido por el diclofenaco (34).

\section{Combinación con posaconazol}

El posaconazol es un triazol de amplio espectro. Actúa inhibiendo la lanosterol 14- $\alpha$ demetilasa, enzima necesaria para la síntesis de ergosterol, por lo que altera la membrana fúngica y el crecimiento celular (38). La estructura química del posaconazol difiere de la del fluconazol y del voriconazol, al interactuar con un dominio adicional de la enzima sobre la que este actúa 
(39), por lo que podría ser efectivo en los hongos resistentes a fluconazol y voriconazol (40). Actualmente es el derivado de azoles más potente y se considera que su combinación con otros grupos de antifúngicos podría tener un efecto terapéutico favorable (41).

Chen y colaboradores (42) evaluaron la combinación del posaconazol con una equinocandina, al estudiar el efecto del posaconazol y la caspofungina en especímenes de C. albicans. El estudio demostró sinergia in vitro entre estos dos antifúngicos, incluso en los especímenes que tenían resistencia a fluconazol o a las equinocandinas. Sin embargo, no mostraron la misma eficacia in vivo sobre especímenes de origen clínico, pues fueron efectivas únicamente contra un espécimen que tenía resistencia a equinocandinas generada mediante ingeniería genética (42).

Combinación con inhibidores de la quitina-sintasa: nicomicina Z

El tratamiento con equinocandinas inhibe la síntesis de $\beta(1,3)$-glucano, polisacárido componente de la pared celular (43). Se ha observado que, in vitro, las equinocandinas estimulan la formación de quitina (44), otro polisacárido de la pared celular (45), el cual reduce la eficacia de las equinocandinas e induce la formación de estructuras nuevas, como septos, que impiden que la célula muera $(46,47)$. Consecuentemente, se ha considerado la posibilidad de combinar las equinocandinas con inhibidores de la quitina-sintasa (44) como estrategia terapéutica frente a infecciones fúngicas.

Walker y colaboradores (44) demostraron que un inhibidor de la quitina-sintasa, la nicomicina Z (figura 1), fue capaz de impedir la formación de septos nuevos in vitro en especímenes de C. albicans y presentó un fuerte efecto sinérgico al combinarse con caspofungina, incluso frente a especímenes de C. albicans con resistencia a equinocandinas.

El potencial de los inhibidores de la quitinasintasa para actuar sobre la pared celular fúngica tiene especial importancia, debido al papel fundamental que la pared celular cumple en la viabilidad de los hongos y su ausencia en las células de los mamíferos (48). Lo anterior hace que esta combinación farmacológica sea potencialmente útil frente a la resistencia a equinocandinas.

Combinación con MGCD290 (inhibidor de la histona-deacetilasa Hos 2)

Las histonas-deacetilasas (HDAC) son enzimas que eliminan el grupo acetilo de los residuos de lisinas en las histonas. Actúan regulando la expresión de genes e influyendo en la proliferación, movilidad y muerte celular (49). Los inhibidores de estas enzimas generan citotoxicidad e inducen la apoptosis celular (49), por lo que se cree que la modulación de la expresión de genes por medio de la inhibición de las HDAC pueden ser una alternativa terapéutica frente a las infecciones fúngicas $(50,51)$.

MGCD290 es un antifúngico nuevo que actúa inhibiendo de la enzima fúngica Hos2, y fue desarrollado por MethylGene, Inc. (Montreal, Quebec, Canadá) (52). Pfaller evaluó su actividad in vitro al combinarlo con azoles, encontrando sinergia con fluconazol frente a $87 \%$ de los especímenes de Candida y actividad frente a muchos de los especímenes resistentes a azoles (51).

Con respecto a la combinación del MGCD290 con las equinocandinas, Pfaller y colaboradores (51) realizaron un estudio in vitro con Candida resistente a equinocandinas. Por medio de 30 especímenes de Candida, se demostró sinergia o sinergia parcial en $30-36,7 \%$ de las muestras tratadas con equinocandinas y MGCD290, la cual fue independiente de la presencia de una mutación en los genes FKS (51).

A pesar de que es un hallazgo alentador e interesante, estudios clínicos de fase II que evaluaron la administración conjunta de fluconazol y MGCD290 en pacientes con vulvovaginitis por Candida no permitieron demostrar la eficacia del MGCD290 (52). 


\section{Combinación con ciclosporina A}

La ciclosporina $\mathrm{A}$ es un producto natural encontrado en hongos que ha demostrado actividad antifúngica. Actúa inhibiendo la actividad de la calcineurina, una fosfatasa que se encuentra tanto en levaduras como en seres humanos (53), con un papel importante en la sobrevida fúngica y en la respuesta frente al estrés (54).

La calcineurina está involucrada en varias funciones fisiológicas de C. albicans incluyendo la morfogénesis, la biosíntesis de la pared, la resistencia antifúngica y la virulencia (55), por lo que los inhibidores de la calcineurina podrían ser útiles en el tratamiento de estas infecciones.

El efecto de la combinación de ciclosporina A con antifúngicos fue evaluado en un estudio in vitro utilizando la cepa estándar de C. albicans (55). Se demostró que al combinar $62,5 \mu \mathrm{g} / \mathrm{ml}$ de ciclosporina $\mathrm{A}$ con caspofungina disminuía la CIM de la caspofungina, e incluso se revirtió la tolerancia en las cepas que presentaban un valor elevado de CIM para caspofungina. Sin embargo, aún faltan estudios in vivo para ampliar el conocimiento sobre la efectividad de la ciclosporina A y su mecanismo de acción como antifúngico (55).

\section{Nuevos compuestos}

Agente E1210: inhibidores de la biosíntesis del glicofosfatidilinositol fúngico

Este agente de amplio espectro (figura 1), conocido por su acción inhibitoria sobre la inositol acetiltransferasa del hongo, altera la maduración de las proteínas ancladas al glicosilfosfatidilinositol (GPI) (56) y, por tanto, impide que actúen como adhesinas (57) para los hongos que entran a las células (58).

Su efectividad en el tratamiento de candidiasis resistente a equinocandinas fue inicialmente demostrada por Pfaller y colaboradores (59), por medio de un estudio in vitro, al encontrar una CIM inferior o igual a $0,12 \mu \mathrm{g} / \mathrm{ml}$ en especímenes de C. albicans, C. glabrata y C. tropicalis, resistentes a caspofungina. Adicionalmente, demostraron que en las anteriores especies de Candida la CIM era mucho menor, comparada con la de fluconazol, posaconazol, voriconazol y caspofungina.

De igual manera, el estudio realizado por Wiederhold y colaboradores (60) comprobó que, además de actuar sobre los especímenes resistentes, también era mejor que la caspofungina sobre los especímenes de tipo silvestre de C. albicans. Como ventaja adicional, este agente permitiría evitar las interacciones medicamentosas, debido a que su mecanismo de acción es únicamente sobre los hongos y no en las células humanas.

Derivado de enfumafungina SCY 078 (previamente conocido como MK 3118)

La enfumafungina es un glucósido triperpenoide aislado de la fermentación de Hormonema sp. que ha mostrado actividad frente a Candida y Aspergillus, al inhibir la $\beta$ - $(1,3)$-glucano-sintasa $(61,62)$.

La actividad del SCY 078, derivado semisintético de la enfumafungina (figura 1), frente a especímenes de Candida resistente a equinocandinas, fue evaluada por JiménezOrtigosa y colaboradores (63). Por medio de un estudio in vitro con 95 especímenes de Candida que incluían las especies albicans, glabrata, dubliniensis, krusei, parapsilosis y tropicalis se demostró que el SCY 078 era activo frente a la mayoría de especímenes con resistencia a equinocandinas mediadas por mutaciones FKS, especialmente frente a C. albicans y C. glabrata. Por ejemplo, la CIM para caspofungina en el 50\% de los especímenes de C. albicans resistentes a equinocandinas fue $\geq 2 \mathrm{mg} / \mathrm{l}$, comparado con la CIM para SCY 078 del $70 \%$ de especímenes, que fue menor de 0,5 mg/l (63).

La actividad de SCY 078 frente a especímenes de Candida con resistencia a equinocandinas con mutaciones FKS conocidas fue confirmada por Pfaller y colaboradores (64), quienes notaron que, además de tener una potencia similar a 
la caspofungina frente a todas las especies de Candida evaluadas, el SCY 078 era 8 veces más potente que la caspofungina frente a los especímenes de C. glabrata (64).

\section{Nueva equinocandina MIG0310}

Descrita por primera vez por Masaphy (65), la MIG0310 es una nueva equinocandina (figura 1) creada con base en la actividad anti-Candida del espécimen del hongo Fusarium brachygibbosum. En un estudio clínico que incluía especímenes clínicos de C. albicans, C. tropicalis, C. krusei y C. glabrata se demostró actividad de MIG0310 frente a los especímenes de C. albicans con CIM $\leq 3,1 \mu \mathrm{g} / \mathrm{ml}(65)$.

A pesar de que actualmente no hay mucha información sobre esta nueva equinocandina, no se descarta que en el futuro pueda utilizarse como una alternativa de tratamiento frente a las infecciones por Candida resistente a equinocandinas.

\section{Nueva equinocandina CD101}

La equinocandina CD101 es un nuevo antifúngico de larga vida media (66). Su mecanismo de acción es similar al de las equinocandinas actualmente aprobadas, el cual es la inhibición de la glucano-sintasa (67) y adicionalmente presenta una modificación en su estructura (figura 1) que le confiere mejores propiedades farmacocinéticas y potencialmente mejor perfil de seguridad en comparación con otros medicamentos de la misma clase $(68,69)$. Este nuevo fármaco ha sido evaluado en soluciones de plasma de rata, mono, perro y humano y ha demostrado mayor estabilidad que la anidulafungina (70). También ha demostrado potente actividad in vitro contra Candida $\mathrm{y}$ Aspergillus, incluyendo algunos especímenes de Candida resistentes a equinocandinas y azoles $(68,71)$.

Por tanto, este nuevo fármaco de amplio espectro ha demostrado tener gran potencial, no solo en el tratamiento de candidiasis invasiva, sino también en el manejo de la emergente resistencia frente a las equinocandinas actuales (68).

\section{Arilamidina T 2307}

La arilamidina T 2307 (figura 1) es un compuesto nuevo (Toyama Chemical Co.) que se está estudiando in vitro e in vivo por su actividad antifúngica de amplio espectro, la cual es especialmente potente contra Candida albicans (72). Además, Wiederhold y colaboradores (73) demostraron que la arilamidina T2307 tiene actividad in vitro contra los especímenes de C. albicans resistentes a equinocandinas (CIM50 $\leq$ $0,008 \mu \mathrm{g} / \mathrm{ml})$. Y en el modelo murino se observó un aumento de la sobrevida y disminución de la cantidad de levaduras presentes en los riñones de los ratones que fueron tratados con T-2307, comparados con aquellos tratados con caspofungina. Este compuesto está actualmente en fase I de estudios clínicos (74).

\section{Otros medicamentos}

\section{Agentes inmunomoduladores}

Entre los agentes inmunomoduladores se encuentran las citocinas, los factores estimulantes de colonias, los anticuerpos y las vacunas. Citocinas como el interferón gamma (INF- $\gamma$ ) son útiles para la prevención y el manejo de infecciones fúngicas como la candidiasis (75). El INF- $\gamma$ potencia los efectos citotóxicos de macrófagos y neutrófilos (76), además de estimular la respuesta de los linfocitos Th1 en las candidiasis invasivas (77). En segundo lugar, los factores estimulantes de colonias, como el factor estimulante de colonias granulocíticas y el factor estimulante de colonias de granulocitos y monocitos, participan en la activación de los neutrófilos potenciando su actividad antifúngica $(78,79)$. Por su parte, los anticuerpos han sido estudiados ampliamente ante la candidiasis invasiva (80). El anticuerpo monoclonal antiHsp 90 inhibe el desarrollo de la resistencia frente a los antifúngicos (81), recibe el nombre 
de Efungumab y actúa al unirse a la proteína Hsp 90, previniendo un cambio conformacional necesario para la viabilidad del hongo (82). El anti-Hsp90 tiene actividad intrínseca contra Candida spp. (83) y exhibe sinergia in vitro al combinarse con caspofungina $(75,84)$.

Por último, las vacunas tienen dos mecanismos de acción: unas generan la activación de Th1 y Th17 (85), lo cual desencadena la respuesta inflamatoria con el reclutamiento de macrófagos, citocinas y neutrófilos en el sitio de infección (86). Este tipo de vacuna se basa en la respuesta inmune del paciente frente al hongo (86). Por otro lado, las vacunas mediadas por anticuerpos actúan por la activación del complemento, neutralizando factores como las adhesinas, e incluso desencadenando directamente la muerte celular fúngica (85).

Los agentes inmunomoduladores han demostrado su efecto al estimular la respuesta inmune frente a las infecciones por Candida en los pacientes inmunocompetentes. Adicionalmente, agentes como las vacunas basadas en la respuesta humoral podrían mejorar el manejo de las infecciones fúngicas en los pacientes inmunocomprometidos, al restaurar su respuesta inmune (85). Por ello, a pesar de que aún se requieren estudios que evalúen su respuesta frente a Candida resistente a equinocandinas, los agentes inmunomoduladores podrían representar una terapia adyuvante al actuar en la respuesta inmune del huésped (75).

\section{Comentarios y conclusión}

Las infecciones por Candida representan una problemática de salud pública, debido a su creciente incidencia y la aparición de especies multirresistentes en el ámbito hospitalario (19). Además, su resistencia frente a las equinocandinas continúa aumentando y el incremento de dosis parece no ser la solución (28), por lo que es indispensable buscar nuevas alternativas terapéuticas, además de establecer estrategias de gerenciamiento de antimicóticos de la misma forma que se recomienda para los antibióticos. En este artículo se revisaron compuestos que se encuentran en estudio, con propiedades antifúngicas frente a especies de Candida resistente a equinocandinas. Sin embargo, la mayoría han sido evaluados únicamente in vitro o en modelos murinos y muy pocos se encuentran en estadios avanzados de investigación. Entre los presentados, cabe resaltar que el agente E1210 (87), la nueva equinocandina CD101 (88) y el SCY078 (MK3118) (89) se encuentran en fase II de estudios clínicos y que el compuesto MGCD 290 fue suspendido durante los estudios de fase II para vulvovaginitis por Candida (90). Adicionalmente, compuestos como el diclofenaco, que ha presentado sinergia con las equinocandinas, generan un interés adicional, debido a que ya se encuentran farmacéuticamente disponibles y con perfiles de seguridad ya establecidos (91).

Estos compuestos, aún novedosos en la literatura médica, representan la oportunidad de generar medicamentos con mejores resultados, que permitan superar la creciente resistencia actual de las infecciones de Candida frente a las equinocandinas.

\section{Referencias}

1. Pappas PG, Kauffman CA, Andes D, Benjamin D K, Calandra TF, Edwards JE, et al. Clinical practice guidelines for the management of candidiasis: 2009 update by the Infectious Diseases Society of America. Clin Infect Dis. 2009;48(5):503-35. doi: 10.1086/596757.

2. Pappas PG, Rex JH, Lee J, Hamill RJ, Larsen RA, Powderly W, et al. A prospective observational study of candidemia: Epidemiology, therapy, influences on mortality in hospitalized adult and pediatric patients. Clin Infect Dis. 2003;37 (5):634-43. doi: 10.1086/376906.

3. Wisplinghoff $\mathrm{H}$, Bischoff $\mathrm{T}$, Tallent SM, Seifert $H$, Wenzel RP, Edmond MB. Nosocomial bloodstream infections in US hospitals: Analysis of 24,179 cases from a prospective nationwide surveillance study. 
Clin Infect Dis. 2004;39(3):309-17. doi: 10.1086/421946.

4. Wey SB, Mori M, Pfaller MA, Woolson RF, Wenzel RP. Hospital-acquired candidemia: The attributable mortality and excess length of stay. Arch Intern Med [Internet]. 1988;148(12):2642-5. Disponible en: https://www.ncbi.nlm.nih.g ov/pubmed/3196127.

5. Voss A, le Noble JL, Verduyn F, Foudraine NA, Meis JF. Candidemia in intensive care unit patients: Risk factors for mortality. Infection 1997;25(1):8-11.

6. Glöckner A, Cornely OA. Practical considerations on current guidelines for the management of non-neutropenic adult patients with Candidaemia. Mycoses [Internet]. 2013;56(1):11-20. Disponible en: https://www.ncbi.nlm.nih.gov/pubmed/ 22574925.

7. Gudlaugsson O, Gillespie S, Lee K, Vande Berg J, Hu J, Messer S, Herwaldt L, Pfaller M, Diekema DL. Attributable mortality of nosocomial candidemia, revisited. Clin Infect Dis 2003;37(9):1172-7.

8. Colombo AL, Nucci M, Park BJ, Nouér SA, Arthington-Skaggs B, da Matta DA, Warnock D, Morgan J; Brazilian Network Candidemia Study. Epidemiology of candidemia in Brazil: A nationwide sentinel surveillance of candidemia in eleven medical centers. J Clin Microbiol. 2006;44(8):2816-23. doi: 10.1128/JCM.00773-06.

9. Pittet D, Wenzel RP. Nosocomial bloodstream infections. Secular trends in rates, mortality and contribution to total hospital deaths. Arch Intern Med [Internet]. 1995;155(11):1177-84. Disponible en: https://www.ncbi.nlm.nih.g ov/pubmed/7763123.

10. Nucci M, Queiroz-Telles F, Tobón AM, Restrepo A, Colombo AL. Epidemiology of opportunistic fungal infections in Latin America. Clin Infect Dis [Internet]. 2010;51(5):561-70. Disponible en: https:// www.ncbi.nlm.nih.gov/pubmed/20658942.
11. Cortés JA, Jaimes JA, Leal AL. Incidence, prevalence of candidemia in critically ill patients in Colombia. Rev Chilena Infectol [Internet]. 2013;30(6):599-604. Disponible en: https://www.ncbi.nlm.nih.g ov/pubmed/24522301.

12. Zaoutis TE, Argon J, Chu J, Berlin JA, Walsh TJ, Feudtner C. The epidemiology and attributable outcomes of candidemia in adults and children hospitalized in the United States: A propensity analysis. Clin Infect Dis [Internet]. 2005;41(9):1232-9. Disponible en: https://www.ncbi.nlm.nih.g ov/pubmed/16206095.

13. Ruhnke M, Eigler A, Tennagen I, Geiseler B, Engelmann E, Trautmann M. Emergence of fluconazole-resistant strains of Candida albicans in patients with recurrent oropharyngeal candidosis and human immunodeficiency virus infection. J Clin Microbiol [Internet]. 1994;32(9):2092-8. Disponible en: https://www.ncbi.nlm.nih.g ov/pubmed/7814530.

14. Beyda ND, Lewis RE, Garey KW. Echinochandin resistance in Candida species: Mechanisms of reduced susceptibility and therapeutic approaches. Ann Pharmacother [Internet]. 2012;46(7-8):1086-96. Disponible en: http s://www.ncbi.nlm.nih.gov/pubmed/228113 50.

15. Food and Drug Administration, Center for Drug Evaluation and Research, Division of Special Pathogen and Immunologic Drug Products. CANCIDAS $^{\mathrm{TM}}$ for invasive aspergillosis: Background document [Internet]. 2001. Disponible en: http://www.fda.gov/ohrms/d ockets/ac/01/briefing/3676b1_01.pdf.

16. U. S. Food and Drug Administration. Drug approval package: Mycamine (Micafungin Sodium) injection [Internet]. 2005. Disponible en http://www.accessdata.fda.gov/drugsatf da_docs/nda/2005/21-506_Mycamine.cfm.

17. U. S. Food and Drug Administration. Package insert for ERAXIS (anidulafungin) for injection [Internet]. Disponible 
en: https://www.accessdata.fda.gov/drugsat fda_docs/label/2006/021632s002lbl.pdf.

18. Glöckner A, Steinbach A, Vehreschild JJ, Cornely OA. Treatment of invasive candidiasis with echinocandins. Mycoses [Internet]. 2009;52(6):476-86. Disponible en: https://www.ncbi.nlm.nih.gov/pubmed/ 19076282.

19. Vallabhaneni S, Kallen A, Tsay S, et al. Investigation of the first seven reported cases of Candida auris, a globally emerging invasive, multidrug-resistant fungus United States, May 2013-August 2016. MMWR Morb Mortal Wkly Rep. ePub: 4 November 2016;16.

20. Morales-López SE, Parra-Giraldo CM, Ceballos-Garzón A, Martínez HP, Rodríguez GJ, Álvarez-Moreno CA, et al. Invasive infections with multidrug-resistant yeast Candida auris, Colombia. Emerg Infect Dis. 2017;23(1):162-4. doi: https:// dx.doi.org/10.3201/eid2301.161497.

21. Dimopoulos G, Antonopoulou A, Armaganidis A, Vincent JL. How to select an antifungal agent in critically ill patients. J Crit Care [Internet]. 2013;28(5):717-27. Disponible en: https://www.ncbi.nlm.nih.g ov/pubmed/2401829.

22. Denning, D. W. Echinocandins: A new class of antifungal. J Antimicrob Chemother [Internet]. 2002;49(6):889-91. Disponible en: https://www.ncbi.nlm.nih.gov/pubmed/ 12039879.

23. Castanheira M, Woosley LN, Diekema DJ, Messer SA, Jones RN, Pfaller MA. Low prevalence of fks1 hot spot 1 mutations in a worldwide collection of Candida strains. Antimicrob Agents Chemother. 2010;54(6):2655-9.

24. Shields RK, Nguyen MH, Press EG, Updike CL, Clancy CJ. Caspofungin MICs correlate with treatment outcomes among patients with Candida glabrata invasive candidiasis and prior echinocandin exposure. Antimicrob Agents Chemother [Internet].2013;57(8):3528-35. Disponible en: https://www.ncbi.nlm.nih.gov/pubmed/ 23669387.
25. Pfaller MA, Castanheira M, Lockhart SR, Ahlquist AM, Messer SA, Jones RN. Frequency of decreased susceptibility and resistance to echinocandins among fluconazoleresistant bloodstream isolates of Candida glabrata. J Clin Microbiol [Internet]. 2012;50(4):1199-203. Disponible en: https ://www.ncbi.nlm.nih.gov/pubmed/2227884 2.

26. Wiederhold NP, Najvar LK, Bocanegra R, Molina D, Olivo M, Graybill JR. In vivo efficacy of anidulafungin and caspofungin against Candida glabrata and association with in vitro potency in the presence of sera. Antimicrob Agents Chemother [Internet]. 2007;51(5):1616-20. Disponible en: https:/ /www.ncbi.nlm.nih.gov/pubmed/1730797 6.

27. Wiederhold NP, Najvar LK, Bocanegra RA, Kirkpatrick WR, Patterson TF. Caspofungin dose escalation for invasive candidiasis due to resistant Candida albicans. Antimicrob Agents Chemother [Internet]. 2011;55(7):3254-60. Disponible en: https:/ /www.ncbi.nlm.nih.gov/pubmed/2150263 2.

28. Domán M, Kovács R, Perlin DS, Kardos G, Gesztelyi R, Juhász B, Bozó A, Majoros L. Dose escalation studies with caspofungin against Candida glabrata. J Med Microbiol [Internet]. 2015;64(9):998-1007. Disponible en: https ://www.ncbi.nlm.nih.gov/pubmed/2629634 0 .

29. Jabra-Rizk MA, Shirtliff M, James C, Meiller T. Effect of Farnesol on Candida dubliniensis biofilm formation and fluconazole resistance. FEMS Yeast Res [Internet] 2006;6(7):1063-73. Disponible en: https://www.ncbi.nlm.nih.gov/pubmed/ 17042756.

30. Hornby JM, Jensen EC, Lisec AD, Tasto JJ, Jahnke B, Shoemaker R, et al. Quorum sensing in the dimorphic fungus Candida albicans is mediated by Farnesol. Appl Environ 
Microbiol [Internet]. 2001;67(7):2982-92. Disponible en: https://www.ncbi.nlm.nih.g ov/pubmed/11425711.

31. Shirtliff ME, Krom BP, Meijering RA, Peters BM, Zhu J, Scheper MA, Harris ML, Jabra-Rizk MA. Farnesol-induced apoptosis in Candida albicans. Antimicrob Agents Chemother [Internet]. 2009;53(6): 2392-401. Disponible en: https://www.ncbi .nlm.nih.gov/pubmed/19364863.

32. Cordeiro RA, Teixeira CE, Brilhante RS, Castelo-Branco DS, Paiva MA, Giffoni Leite JJ, Lima DT, Monteiro AJ, Sidrim JJ, Rocha MF. Minimum inhibitory concentrations of amphotericin B, azoles and caspofungin against Candida species are reduced by Farnesol. Med Mycol [Internet]. 2013;51(1):53-9. Disponible en: https://www.ncbi.nlm.nih.gov/pubmed/ 22712455.

33. Alem MA, Douglas LJ. Prostaglandins in production during growth of Candida albicans biofilms. J Med Microbiol. 2005;54(11):1001-5.

34. Bink A, Kucharíková S, Neirinck B, Vleugels J, Van Dijck P, Cammue BP, Thevissen $\mathrm{K}$. The nonsteroidal antiinflammatory drug diclofenac potentiates the in vivo activity of caspofungin against Candida albicans biofilms. J Infect Dis. 2012;206 (11):1790-7.

35. Vane JR, Botting R. M. Mechanism of action of nonsteroidal antiinflammatory drugs. Am J Med [Internet]. 1998;104(3A)2S-8S; discussion 21S-22S. Disponible en: https://www.ncbi.nlm.nih.g ov/pubmed/9572314.

36. Noverr MC, Phare SM, Toews GB, Coffey MJ, Huffnagle G. B. Pathogenic yeasts Cryptococcus neoformans and Candida albicans produce immunomodulatory prostaglandins. Infect Immun [Internet]. 2001;69(5):2957-63. Disponible en: https://www.ncbi.nlm.nih.g ov/pubmed/11292712.

37. Erb-Downward JR, Noverr MC. Characterization of prostaglandin E2 production by Candida albicans. Infect
Immun [Internet]. 2007;75(7):3498-505. Disponible en: https://www.ncbi.nlm.nih.g ov/pubmed/17470538.

38. Nagappan V, Deresinski S. Reviews of antiinfective agents: posaconazole: A broadspectrum triazole antifungal agent. Clin Infect Dis [Internet]. 2007;45(12):1610-7. Disponible en: https://www.ncbi.nlm.nih.g ov/pubmed/18190324.

39. Xiao L, Madison V, Chau AS, Loebenberg D, Palermo RE, McNicholas PM. Three-dimensional models of wildtype and mutated forms of cytochrome P450 14alpha-sterol demethylases from Aspergillus fumigatus and Candida albicans provide insights into posaconazole binding. Antimicrob Agents Chemother [Internet]. 2004;48(2):568-74. Disponible en: https:// www.ncbi.nlm.nih.gov/pubmed/14742211.

40. Chau AS, Mendrick CA, Sabatelli FJ, Loebenberg D, McNicholas PM. Application of real-time quantitative PCR to molecular analysis of Candida albicans strains exhibiting reduced susceptibility to azoles. Antimicrob Agents Chemother. 2004;48(6):2124-31.

41. Hof H. A new, broad-spectrum azole antifungal: posaconazole-mechanisms of action and resistance, spectrum of activity. Mycoses [Internet]. 2006;49 Suppl 1:2-6. Disponible en: https://www.ncbi.nlm.nih.g ov/pubmed/16961575.

42. Chen YL, Lehman VN, Averette AF, Perfect JR, Heitman J. Posaconazole exhibits in vitro and in vivo synergistic antifungal activity with caspofungin or FK506 against Candida albicans. PLoS One. 2013;8(3):e57672.

43. Sucher AJ, Chahine EB, Balcer $H$. E. Echinocandins: the newest class of antifungals. Ann Pharmacother [Internet]. 2009;43(10):1647-57. Disponible en: https ://www.ncbi.nlm.nih.gov/pubmed/1972401 4.

44. Walker LA, Munro CA, de Bruijn I, Lenardon MD, McKinnon A, Gow NA. Stimulation of chitin synthesis rescues Candida albicans from echinocandins. 
PLoS Pathog [Internet]. 2008;4(4). Disponible en: https:/www.ncbi.nlm.nih.g ov/pubmed/18389063.

45. Klis FM, De Groot P, Hellingwerf K. Molecular organization of the cell wall of Candida albicans. Med Mycol [Internet]. 2001;39 Suppl 1:1-8. Disponible en: https:// www.ncbi.nlm.nih.gov/pubmed/11800263.

46. Walker LA, Gow NA, Munro CA. Elevated chitin content reduces the susceptibility of Candida species to caspofungin. Antimicrob Agents Chemother [Internet]. 2013;57(1):146-54. Disponible en: https:// www.ncbi.nlm.nih.gov/pubmed/23089748.

47. Lenardon MD, Munro CA, Gow NA. Chitin synthesis and fungal pathogenesis. Curr Opin Microbiol [Internet]. 2010;13(4):416-23. Disponible en: https://www.ncbi.nlm.nih.gov/pubmed/ 20561815.

48. Escobar C. Nuevos antimicóticos y su uso en dermatología. Med Cut Iber Lat Am. 2004;32(6):231-42.

49. De Ruijter AJ, Van Gennip AH, Caron HN, Kemp S, Van Kuilenburg AB. Histone deacetylases (HDACs): Characterization of the classical HDAC family. Biochem J [Internet]. 2003;370(3):737-49. Disponible en: https://www.ncbi.nlm.nih.g ov/pubmed/12429021.

50. Smith WL, Edlind TD. Histone deacetylase inhibitors enhance Candida albicans sensitivity to azoles and related antifungals: correlation with reduction in CDR and ERG upregulation. Antimicrob Agents Chemother [Internet]. 2002;46(11):3532-9. Disponible en: https:/ /www.ncbi.nlm.nih.gov/pubmed/1238436 1.

51. Pfaller MA, Rhomberg PR, Messer SA, Castanheira M. In vitro activity of a Hos2 deacetylase inhibitor, MGCD290, in combination with echinocandins against echinocandin-resistant Candida species. Diagn Microbiol Infect Dis [Internet]. 2015;81(4):259-63. Disponible en: https:// www.ncbi.nlm.nih.gov/pubmed/25600842.
52. Mirati Therapeutics. MethylGene reports results of phase II Trial of MGCD290 [Internet]. Disponible en: http://ir.mirati.c om/releasedetail.cfm? releaseid $=787643$.

53. Rusnak F, Mertz P. Calcineurin: form and function. Physiol Rev [Internet]. 2000;80(4):1483-521. Disponible en: https://www.ncbi.nlm.nih.g ov/pubmed/11015619.

54. Li Y, Sun S, Guo Q, Ma L, Sh, C, Su $\mathrm{L}$, Li H. In vitro interaction between azoles and cyclosporin A against clinical isolates of Candida albicans determined by the chequerboard method, timekill curves. J Antimicrob Chemother [Internet]. 2008;61(3):577-85. Disponible en: https://www.ncbi.nlm.nih.gov/pubmed/ 18194958.

55. Shinde RB, Chauhan NM, Raut JS, Karuppayil SM. Sensitization of Candida albicans biofilms to various antifungal drugs by cyclosporine A. Ann Clin Microbiol Antimicrob [Internet]. 2012;11(27). Disponible en: https://www.n cbi.nlm.nih.gov/pubmed/23035934.

56. Miyazaki M, Horii T, Hata K, Watanabe NA, Nakamoto K, Tanaka K, Shirotori $S$, Murai $\mathrm{N}$, Inoue $\mathrm{S}$, Matsukura $\mathrm{M}$, Abe S, Yoshimatsu K, Asada M. In vitro activity of E1210, a novel antifungal, against clinically important yeasts, molds. Antimicrob Agents Chemother [Internet]. 2011;55(10):4652-8. Disponible en: https:/ /www.ncbi.nlm.nih.gov/pubmed/2182529 1.

57. Chaffin WL. Candida albicans cell wall proteins. Microbiol Mol Biol Rev. 2008;72(3):495-544.

58. Sheppard DC, Yeaman MR, Welch WH, Phan QT, Fu Y, Ibrahim AS, Filler SG, Zhang M, Waring AJ, Edwards JE. Functional and structural diversity in the Als protein family of Candida albicans. J Biol Chem [Internet]. 2004;279(29):30480-9. Disponible en: https://www.ncbi.nlm.nih.g ov/pubmed/15128742.

59. Pfaller MA, Hata K, Jones RN, Messer SA, Moet GJ, Castanheira M. In vitro activity of 
a novel broad-spectrum antifungal, E1210, tested against Candida spp. as determined by CLSI broth microdilution method. Diagn Microbiol Infect Dis [Internet]. 2011;71(2):167-70. Disponible en: https:// www.ncbi.nlm.nih.gov/pubmed/21696907.

60. Wiederhold NP, Najvar LK, Fothergill AW, McCarthy DI, Bocanegra R, Olivo M, Kirkpatrick WR, Everson MP, Duncanson FP, Patterson TF. The investigational agent E1210 is effective in treatment of experimental invasive candidiasis caused by resistant Candida albicans. Antimicrob Agents Chemother [Internet]. 2015;59(1): 690-2. Disponible en: https://www.ncbi.nl m.nih.gov/pubmed/25331706.

61. Peláez F, Cabello A, Platas G, Díez MT, González del Val A, Basilio A, Martán I, et al. The discovery of enfumafungin, a novel antifungal compound produced by an endophytic Hormonema species biological activity and taxonomy of the producing organisms. Syst Appl Microbiol [Internet]. 2000;23(3):333-43. Disponible en: https:// www.ncbi.nlm.nih.gov/pubmed/11108011.

62. Onishi J, Meinz M, Thompson J, Curotto J, Dreikorn S, Rosenbach M, et al. Discovery of novel antifungal (1,3)- $\beta$-D-glucan synthase inhibitors. Antimicrob Agents Chemother [Internet]. 2000;44(2):368-77. Disponible en: https://www.ncbi.nlm.nih.g ov/pubmed/10639364.

63. Jiménez-Ortigosa C, Paderu P, Motyl MR, Perlin D. S. Enfumafungin derivative MK-3118 shows increased in vitro potency against clinical echinocandin-resistant Candida Species and Aspergillus species isolates. Antimicrob Agents Chemother [Internet]. 2014;58(2):1248-51. Disponible en: https:/ /www.ncbi.nlm.nih.gov/pubmed/2432347 2.

64. Pfaller MA, Messer SA, Motyl MR, Jones RN, Castanheira M. Activity of MK-3118, a new oral glucan synthase inhibitor, tested against Candida spp. by two international methods (CLSI and EUCAST). J Antimicrob Chemother
[Internet]. 2013;68(4):858-63. Disponible en: https://www.ncbi.nlm.nih.gov/pubmed/ 23190764.

65. Masaphy S. A novel echinocandin MIG0310 with anticandida activity from newly isolated Fusarium sp. strain MS-R1. J Appl Microbiol [Internet]. 2014;116(6):1458-64. Disponible en: https ://www.ncbi.nlm.nih.gov/pubmed/2467445 0.

66. Locke J, Almaguer A, Zuill D, Bartizal $\mathrm{K}$. Characterization of in vitro resistance development to the novel echinocandin, CD101, in Candida species. Antimicrob Agents Chemother. 2016;60(10):6100-7. doi: 10.1128/AAC.00620-16.

67. Pfaller M, Messer S, Rhomberg P, Jones R, Castanheira M. Activity of a longacting echinocandin, CD101, determined using CLSI and EUCAST reference methods, against Candida and Aspergillus spp., including echinocandin-and azoleresistant isolates. J Antimicrob Chemother. 2016;71(10):2868-73. doi: $10.1093 / \mathrm{jac} /$ dkw214.

68. Zhao Y, Perez WB, Jiménez-Ortigosa C, Hough G, Locke JB, Ong V, Bartizal K, Perlin DS. CD101: a novel long-acting echinocandin. Cell Microbiol. 2016;18(9):1308-16. doi: 10.1111/cmi.12640.

69. Ong V, Miesel L, Bartizal K, Huang HH, Chien JC. Prolonged efficacy following one dose of a novel echinocandin, CD101, in a neutropenic mouse model of disseminated candidiasis [Internet]. San Diego: Cidara Therapeutics; s. f. Disponible en: http://www.eurofins.com/media/31367 3/eurofins_cidera.pdf.

70. Krishnan BR, James KD, Polowy K, Bryant BJ, Vaidya A, Smiths S, Laudeman CP. CD101, a novel echinocandin with exceptional stability properties and enhanced aqueous solubility. J Antibiot (Tokyo). 2017;70(2):130-5. doi: 10.1038/ ja.2016.89.

71. Castanheira M, Messer SA, Rhomberg PR, Jones RN, Pfaller MA. Activity of 
a novel echinocandin biafungin (CD101) tested against most common Candida and Aspergillus species, including echinocandinand azole-resistant strains. M-1082 ICAAC 2014 JMI Laboratories North Liberty, IA, USA.

72. Nishikawa $H$, Yamada E, Shibata $T$, Uchihashi S, Fan H, Hayakawa H, Nomura $\mathrm{N}$, Mitsuyama J. Uptake of T-2307, a novel arylamidine, in Candida albicans. J Antimicrob Chemother [Internet]. 2010;65(8): 1681-7. Disponible en: https:// www.ncbi.nlm.nih.gov/pubmed/20513704.

73. Wiederhold NP, Najvar LK, Fothergill AW, Bocanegra R, Olivo M, McCarthy DI, Kirkpatrick WR, Fukuda Y, Mitsuyama J, Patterson T F. The novel arylamidine $\mathrm{T}-2307$ maintains in vitro and in vivo activity against echinocandin-resistant Candida albicans. Antimicrob Agents Chemother [Internet]. 2015;59(2):1341-3. Disponible en: https://www.ncbi.nlm.nih.g ov/pubmed/25451054.

74. T-2307 Antifungal agent Phase 1 [Internet]. Disponible en: http://www.toyama-chemic al.co.jp/en/rd/pipeline/index.htm.

75. Beyda N, Regen S, Lewis R, Garey K. Immunomodulatory agents as adjunctive therapy for the treatment of resistant Candida species. Current Fungal Infection Reports. 2013;7(2):119-25.

76. Hübel K, Dale DC, Liles W. C. Therapeutic use of cytokines to modulate phagocyte function for the treatment of infectious diseases: current status of granulocyte colony-stimulating factor, granulocytemacrophage colony-stimulating factor, macrophage colony-stimulating factor and interferon-gamma. J Infect Dis [Internet]. 2002;185(10):1490-501. Disponible en: https://www.ncbi.nlm.nih.g ov/pubmed/11992286.

77. Gozalbo D, Maneu V, Gil ML. Role of IFN-gamma in immune responses to Candida albicans infections. Front Biosci (Landmark Ed) [Internet]. 2014;19:1279-90. Disponible en: https://w ww.ncbi.nlm.nih.gov/pubmed/24896350.
78. Netea MG, Van der Meer JW, Van Deuren M, Kullberg B. J. Proinflammatory cytokines and sepsis syndrome: not enough, or too much of a good thing? Trends Immunol [Internet]. 2003;24(5):254-8. Disponible en: https://www.ncbi.nlm.nih.gov/pubmed/ 12738419.

79. Djeu JY, Liu, JH, Wei, S, Rui, H, Pearson CA, Leonard WJ, Blanchard DK. Function associated with IL-2 receptor-beta on human neutrophils: Mechanism of activation of antifungal activity against Candida albicans by IL-2. J Immunol [Internet]. 1993;150(3):960-70. Disponible en: https://www.ncbi.nlm.nih.g ov/pubmed/8380826.

80. Bugli F, Cacaci M, Martini C, Torelli R, Posteraro B, Sanguinetti M, Paroni Sterbini F. Human monoclonal antibodybased therapy in the treatment of invasive candidiasis. Clin Dev Immunol. 2013;403121. doi: 10.1155/2013/403121.

81. Cowen LE, Singh SD, Köhler JR, Collins C, Zaas AK, Schell WA, et al. Harnessing Hsp90 function as a powerful, broadly effective therapeutic strategy for fungal infectious disease. Proc Natl Acad Sci U S A [Internet]. 2009;106(8):2818-23. Disponible en: https://www.ncbi.nlm.nih.g ov/pubmed/19196973.

82. Karwa R, Wargo KA. Efungumab: a novel agent in the treatment of invasive candidiasis. Ann Pharmacother [Internet]. 2009;43(11):1818-23. Disponible en: https ://www.ncbi.nlm.nih.gov/pubmed/1977352 8.

83. Matthews RC, Rigg G, Hodgetts S, Carter T, Chapman C, Gregory C, Illidge C, Burnie J. Preclinical assessment of the efficacy of mycograb, a human recombinant antibody against fungal HSP90. Antimicrob Agents Chemother [Internet]. 2003;47 (7):2208-16. Disponible en: https:/ /www.ncbi.nlm.nih.gov/pubmed/1282147 0.

84. Hodgetts S, Nooney L, Al-Akeel R, Curry A, Awad S, Matthews R, Burnie J. Efungumab and caspofungin: pre-clinical 
data supporting synergy. J Antimicrob Chemother [Internet]. 2008;61(5):1132-9. Disponible en: https://www.ncbi.nlm.nih.g ov/pubmed/18299636.

85. Cassone A, Casadevall A. Recent progress in vaccines against fungal diseases. Curr Opin Microbiol. 2012;15(4):27-33. doi: 10.1016/ j.mib.2012.04.004.

86. Kumar P, Chen K, Kolls JK. Th17 cell based vaccines in mucosal immunity. Curr Opin Immunol [Internet]. 2013;25(3):373-80. Disponible en: https://www.ncbi.nlm.nih.g ov/pubmed/23669353.

87. Spampinato C, Leonardi D. Candida infections, causes, targets, and resistance mechanisms: traditional and alternative antifungal agents. Biomed Res Int [Internet]. 2013;204237. Disponible en: https://www.ncbi.nlm.nih.gov/pubmed/ 23878798.

88. Cidara Therapeutics. Rezafungin (CD101 IV) overview [Internet]. Disponible en: htt p://www.cidara.com/cd101-iv/.

89. SCYNEXIS. SCYNEXIS, Inc. initiates enrollment of the phase 2 study of SCY-078 in vulvovaginal candidiasis [Internet]. Jersey City; 2015. Disponible en: http://ir.scynexis.com/news-releases/ne ws-release-details/scynexis-inc-initiates-en rollment-phase-2-study-scy-078.

90. Adis Insight. MGCD 290: Drug profile [Internet]. Disponible en: http://adisinsigh t.springer.com/drugs/800018871

91. Nett J. Future directions for anti-biofilm therapeutics targeting Candida. Expert Rev. Anti Infect Ther. 2014;12(3):375-82. doi: $10.1586 / 14787210.2014 .885838$. 